\title{
Trabalhando a Física Moderna em um projeto de Iniciação Científica Júnior: A experimentação da medida da constante de Planck no Ensino Médio
}

\author{
Yuri Alexandre Meyer ${ }^{2}$ \\ Luis Fernando de Avila ${ }^{3}$ \\ Marina Feliciano Orlandini ${ }^{4}$ \\ Joel Bacellar Neves ${ }^{5}$
}

\section{Resumo}

Este trabalho foi concebido como uma Iniciação Científica Júnior (ICJ), realizado com alunos do Ensino Médio. Foi proposta a construção de um aparato experimental de baixo custo e fácil reprodutibilidade para o estudo da Constante de Planck, através de um circuito com fotorresistor (LDR) e LEDS de diferentes cores (amarelo, azul, vermelho e violeta). Inicialmente são apresentados os aspectos teóricos da Equação de Planck, a qual deu início à Física Moderna no ano de 1900. Para realização da parte experimental os alunos foram divididos em três grupos, e os resultados apresentados estão em boa concordância com os valores encontrados na literatura. Como forma de análise de aprendizagem, além da verificação experimental da constante obtida, foi realizada uma avaliação diagnóstica aplicando quatro questões de vestibulares aos alunos da ICJ e aos alunos do terceiro ano (os quais não tiveram contato com este trabalho). O objetivo central é permitir o desenvolvimento experimental de um tema de grande relevância e o reforço da necessidade da inserção da Física Moderna no ensino médio, uma vez que a difículdade em discutir este tema é muito alta.

Palavras-chave: Física Moderna. Constante de Planck. Iniciação Científica Júnior.

\begin{abstract}
This work is a Scientific Junior Initiation (SJC) and it was accomplished with High School students. The researchers proposed the construction of a experimental display of low cost and easy reproducibility for the study of Planck's Constant, through an electric circuit with photoresistor (LDR) and LEDs of different colors (yellow, blue, red and violet). Initially, the article presents the theoretical aspects of Planck's Equation, which gave birth to Modern Physics in the year of 1900. To the accomplishment of the experimental part, the students were divided into three groups, and the results presented are very close to the theory's expected value. As a form of learning analysis, besides the experimental verification of the obtained constant, the group performed a diagnostic analysis applying four vestibular questions to the senior students (who did not have contact with this work). The central objective of the article is to permit the experimental development of a highly relevant theme and the restatement of the need of inserting Modern Physics in High School, now that the vestibulares are assessing this theme.
\end{abstract}

Keywords: Modern Physics. Plancks Constant. Scientific Junior Initiation.

\footnotetext{
${ }^{1}$ Também participaram da autoria deste artigo: Eduardo Schultz Pilon; Enrico Müller Alves de Mello; Felipe Marmo Miguel; Leonardo Consentino Braga; Leonardo dos Santos Dapolito; Maria Carolina Cardoso Grochoski; Mell de Barros Bergamasco; Raíssa de Sampaio Barros; Rebeca Peixoto Rossini Borges; Vitor Hugo Degan.

${ }^{2}$ Doutorando em Tecnologia, na área de Ciência dos Materiais da Faculdade de Tecnologia da Universidade Estadual de Campinas (UNICAMP). E-mail: meyeryuri@gmail.com

${ }^{3}$ Doutor em Física, é docente na Universidade Estadual de Campinas (UNICAMP). E-mail: luisfdeavila@gmail.com

${ }^{4}$ Graduanda em Medicina pela Faculdade de Ciências Médicas de Santos.

${ }^{5}$ Colégio Anglo Portal de Limeira.
} 
Introdução

Um dos maiores desafios do ensino de Física é mostrar aos alunos a importância desta Ciência em suas vidas. Muitos alunos indagam seus professores: mas por que tenho que saber isso? Não vou usar isso em minha vida! São perguntas frequentes numa sala de aula, as quais, acima de tudo, podem refletir um regresso no processo de conscientização social em relação à área científica, uma vez que as aulas são centradas num currículo clássico de divisões conceituais - muitos dos quais são não articulados, mas fragmentados. Tornam-se então desinteressantes para muitos alunos, uma vez que se baseia apenas no processo de decorar e aplicar fórmulas. Conforme destaca Oliveira, Vianna, e Gerbassi (2007, p. 448),

\begin{abstract}
A lacuna provocada por um currículo de física desatualizado resulta numa prática pedagógica desvinculada e descontextualizada da realidade do aluno. Isso não permite que ele compreenda qual a necessidade de se estudar essa disciplina que, na maioria dos casos, se resume em aulas baseadas em fórmulas e equações matemáticas, excluindo o papel histórico, cultural e social que a física desempenha no mundo em que vive.
\end{abstract}

Assim, os professores de Física têm como grande desafio ensinar um conteúdo que contemple e valorize a formação de um cidadão consciente das mudanças sociais e tecnológicas ao seu redor, despertando o interesse dos discentes. Deste modo, devemos ter em mente que na Física as grandes descobertas que resultaram em aplicações de suma importância para a sociedade aconteceram, em sua grande maioria, com o desenvolvimento da Física Moderna, e os historiadores da Ciência atribuem como sendo o pai da Física Moderna o físico alemão Max Planck, quando em Dezembro de 1900 apresentou seus estudos sobre a radiação do corpo negro para a Sociedade Alemã de Física, propondo então a quantização da energia. Devemos então nos atentar para a necessidade da inserção do ensino da Física Moderna no Ensino Médio, já que

A deficiência no ensino de Física Moderna causa certos prejuízos na formação dos estudantes como:
o não ensino de muitos conhecimentos necessários para a compreensão de muitos dos aspectos
tecnológicos do mundo contemporâneo como às telecomunicações com e sem fio, a aplicação da
Física na área da medicina, engenharias, funcionamento de aparatos tecnológicos de uso diário como
smartphones, computadores, CD'S, DVD'S, internet, etc. (CÉSAR; OLIVEIRA; PAIVA, 2016, p. 2).

Ou ainda,

Em nosso cotidiano estamos cercados por tecnologia: televisões com alta definição, câmeras digitais, laser, leitor de código de barras, raios-X e celulares. Nos meios de comunicação é comum ver notícias de novas descobertas científicas ou aparatos tecnológicos, porém estas informações não são facilmente compreendidas por grande parte da população. (CHICÓRA; CAMARGO; TOPPEL, 2015, p. 20176).

É certo que o currículo de Física está centrado em um processo de conteúdo engessado na essência da Física Clássica, mas não podemos deixar de lado os ensinamentos sobre as aplicações das pesquisas científicas no desenvolvimento tecnológico (e consequentemente social), muitos dos quais oriundos do desenvolvimento da 
Física Moderna. Assim, pode-se dizer que, em partes, o ensino está defasado das realidades sociais e do progresso científico.

Por outro lado não podemos nos esquecer de que o ensino médio é a fase de ajudar os alunos a despertarem suas vocações. Os professores são, portanto, agentes formadores de recursos humanos. Se tomarmos estatísticas de relações candidatos vagas em cursos superiores não é difícil concluir que as carreiras científicas são aquelas com menores índices de interessados. Talvez um dos fatores que contribua para esta questão seja a prática de um ensino metódico que se preocupa apenas com o cumprimento dos muitos tópicos ao longo dos três anos do ensino médio, focando apenas em informações, deixando-se de lado a essência do conhecimento prático e socialmente aplicável. É importante sermos agentes modificadores. Este trabalho procura então mostrar a importância de abordar a Física Moderna no Ensino Médio como meio de incentivo no processo de formação dos alunos, fazendo despertar o interesse pelas questões científicas. Através de um projeto de iniciação científica realizado ao longo de 2018 desenvolvemos uma metodologia experimental para determinar a constante de Planck. São apresentados dois resultados neste trabalho: a obtenção da constante de Planck por um método de fácil reprodutibilidade e uma avaliação diagnóstica com questões de vestibulares, sobre o tema proposto, comparando os índices de acertos dos alunos envolvidos neste projeto com alunos que não tiveram contato com a Iniciação Científica Júnior.

\section{Aspectos teóricos}

A equação de Planck é descrita por:

$$
E=h f(1)
$$

Onde:

$E$ corresponde a energia, $f$ é a frequência da luz e $h$ corresponde a constante de Planck, cujo valor é de $6,62607004 \times 10^{-34} \mathrm{~J} . \mathrm{s}^{-1}$

Podemos reescrever a frequência em termos do comprimento de onda e da velocidade da luz, isto é:

$$
f=\frac{c}{\lambda}
$$

Em que $c$ corresponde a velocidade da luz no vácuo, cujo valor é de $299792458 \mathrm{~m} / \mathrm{s}$.

Assim, substituindo (2) em (1):

$$
E=\frac{h c}{\lambda}
$$

Mas, por sua vez, podemos inferir que:

$$
E=e V
$$

Sendo $e$ a carga fundamental do elétron, cujo valor corresponde a $1,6 \times 10^{-19} \mathrm{C}$ e $V$ é a tensão em Volts.

Deste modo, substituindo (4) em (3): 


$$
e V=\frac{h c}{\lambda}
$$

Rearranjando a equação anterior:

$$
V=\frac{h c}{e} \frac{1}{\lambda}
$$

Onde podemos notar que $\frac{h c}{e}$ corresponde ao coeficiente angular $(m)$ do gráfico de $V$ em função de $1 / \lambda$ . Assim, a constante de Planck poderá ser determinada do seguinte modo:

$$
h=\frac{m e}{c}
$$

\section{Metodologia}

O experimento em questão foi realizado seguindo os mesmos moldes de uma Iniciação Científica praticada nas universidades, isto é, estudo das referências, experimentação, análise e discussão de resultado e redação do artigo científico. Todas as etapas anteriores foram adaptadas à realidade dos alunos do ensino médio. Assim, foram realizadas reuniões periódicas com os alunos (a cada duas semanas) para orientação do embasamento teórico discussões das referências e teorias, e a montagem do experimento.

\section{Figura 1 - Circuito montado para as medições. Nota-se que se deve intercalar as medidas dos LEDS.}

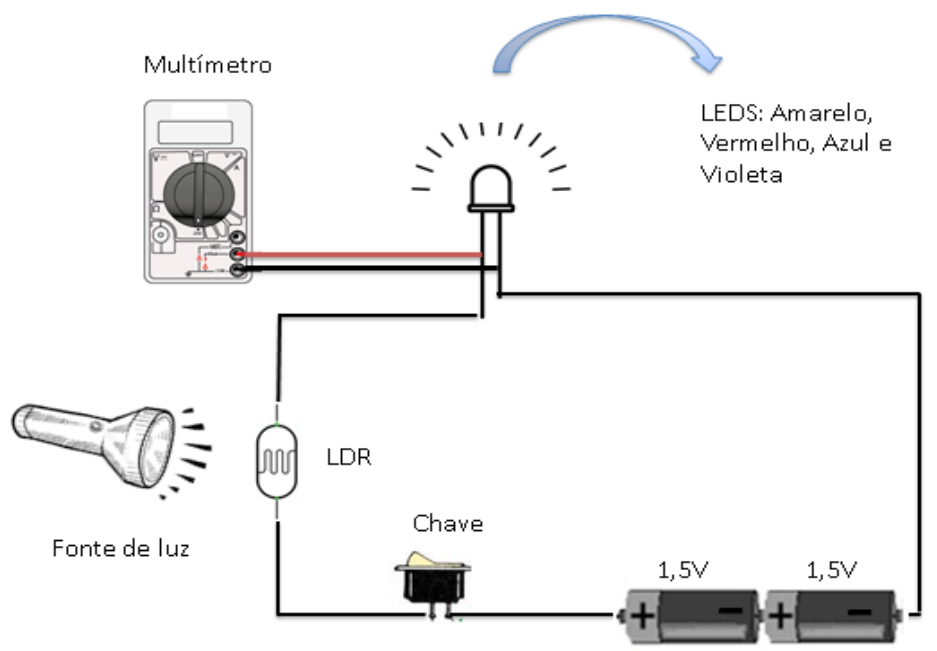

Fonte: dos autores, 2018.

A medida da constante de Planck utilizando LEDS (Light Emitting Diode) é descrita por Amorim, André, André (2014, p.29 - 32) através da construção de um circuito que utiliza um potenciômetro. No entanto, resolvemos testar o circuito já demonstrado em trabalho anterior (Meyer et. al, 2018), em que propusemos, na ocasião, a 


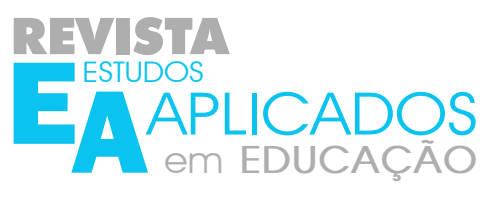

montagem de um circuito com bateria, chave, fotorresistor LDR (Light Dependent Resistor) e LED. Neste circuito os componentes (duas pilhas de 1,5V ligadas em série, chave, fotorresistor e LEDS) são conectados em série conforme é destacado na Figura 1.

Vale ressaltar que o circuito deve ser colocado dentro uma caixa de MDF (tamanho pequeno), com orifício de abertura para uma fonte de luz (lanterna, por exemplo). As leituras de tensões são efetuadas com um multímetro.

Para efetuar as medidas intercalamos para cada leitura uma cor de LED. Nosso objetivo é mostrar que esse circuito, além de servir para experimento da Lei de Lambert-Beer, retratado em trabalho anterior (Meyer et. al., 2018, p. 63), também serve para demonstrar a medida da constante de Planck. Os alunos foram divididos em três grupos, sendo que cada grupo montou o seu próprio circuito. Resolvemos dividi-los em grupos a fim termos uma triplicata dos resultados obtidos. Foram usados quatro cores de LEDS: amarelo, azul, vermelho e violeta. Para cada LED foi sugerido que os alunos fizessem 5 medidas, a fim de calcular uma média. A obtenção dos valores das tensões sobre os LEDS é obtida quando ilumina-se o LDR com auxílio de uma lanterna. O valor experimental da constante de Planck é encontrada mediante a elaboração de um gráfico de tensão $V$ versus inverso do comprimento de onda $1 / \lambda$, obtidos pela média dos valores dos comprimentos expressos na Tabela 1 no Anexo 1. Através dos gráficos plotados, extraem-se os coeficientes angulares e, substituindo-os na equação (7), é possível calcular a constante de Planck. Os resultados são apresentados na próxima seção. Abaixo são expostas duas fotos do procedimento experimental.

Figura 2 - Experimento montado - parte interna.

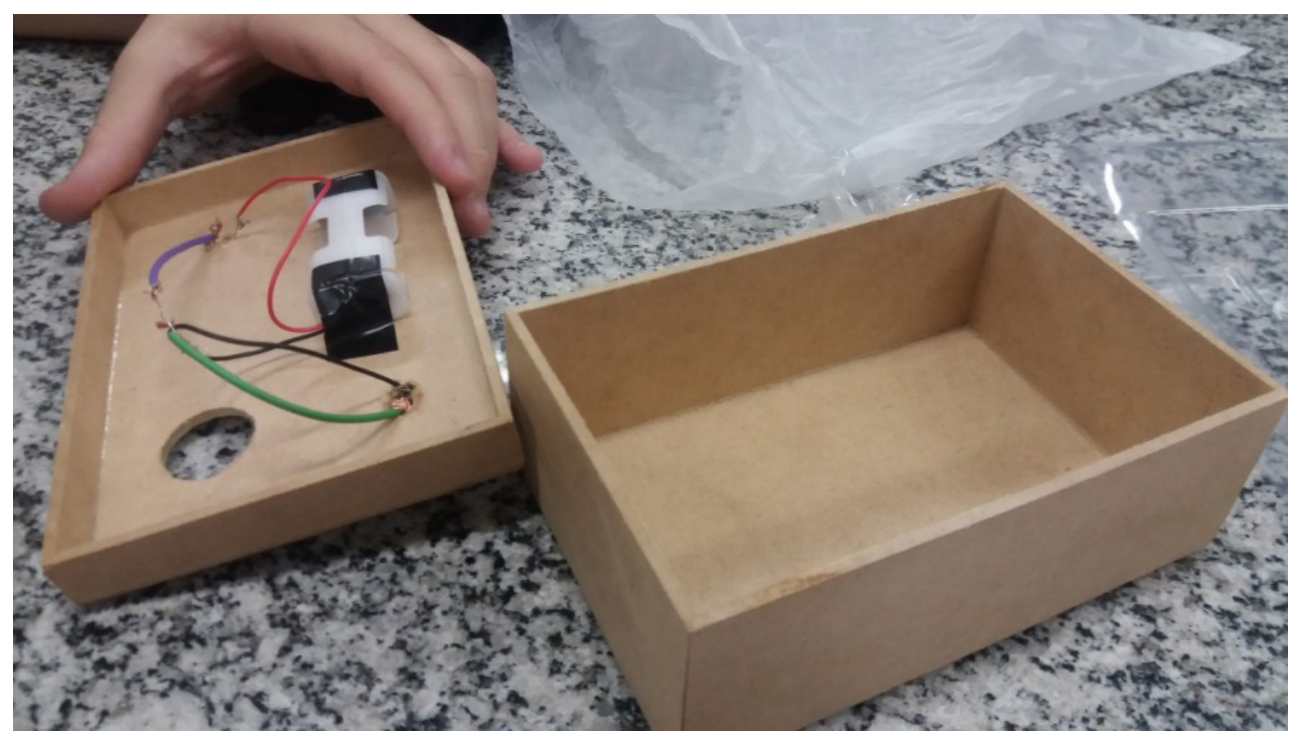

Fonte: dos autores, 2018.

Figura 3 - Experimento montado - parte externa 


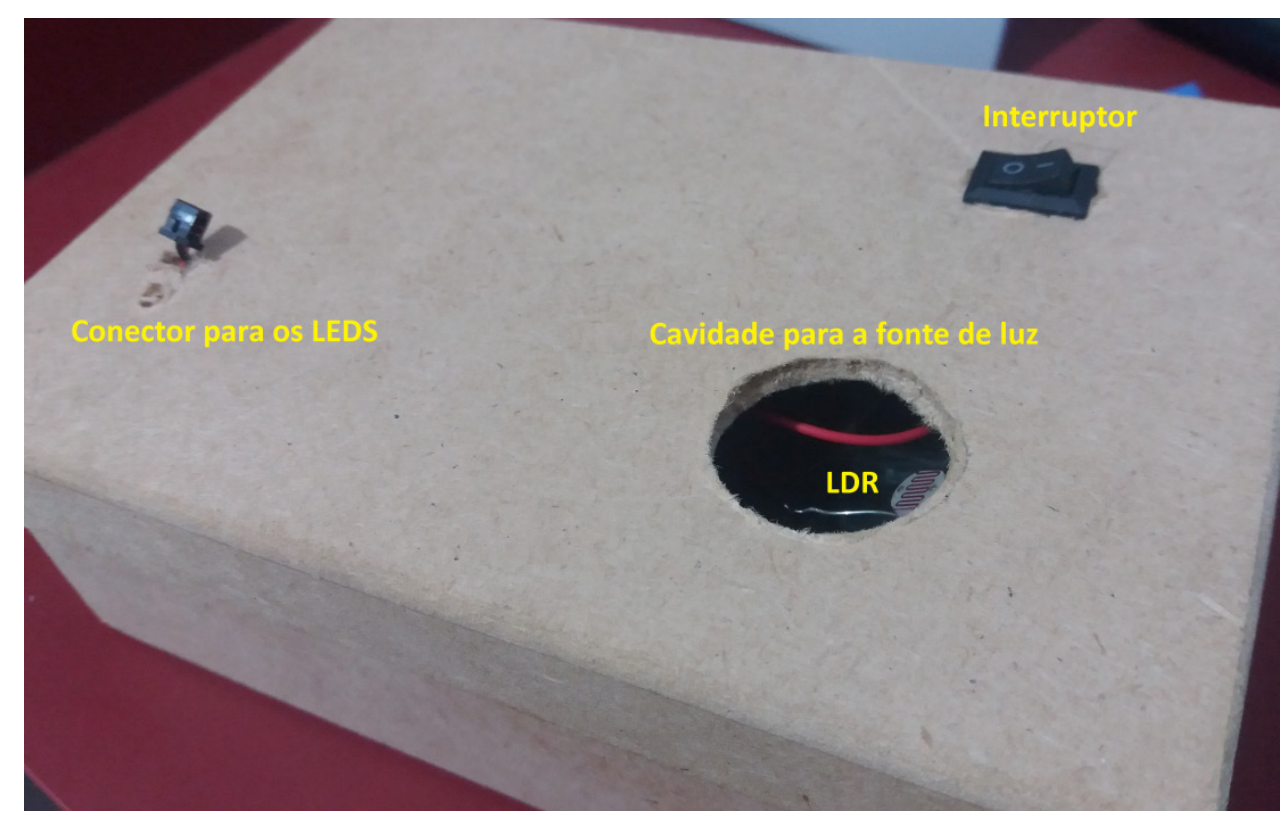

Fonte: dos autores, 2018.

Figura 4 - Aluno fazendo teste com a lanterna do celular. Observa-se que o LED acende.

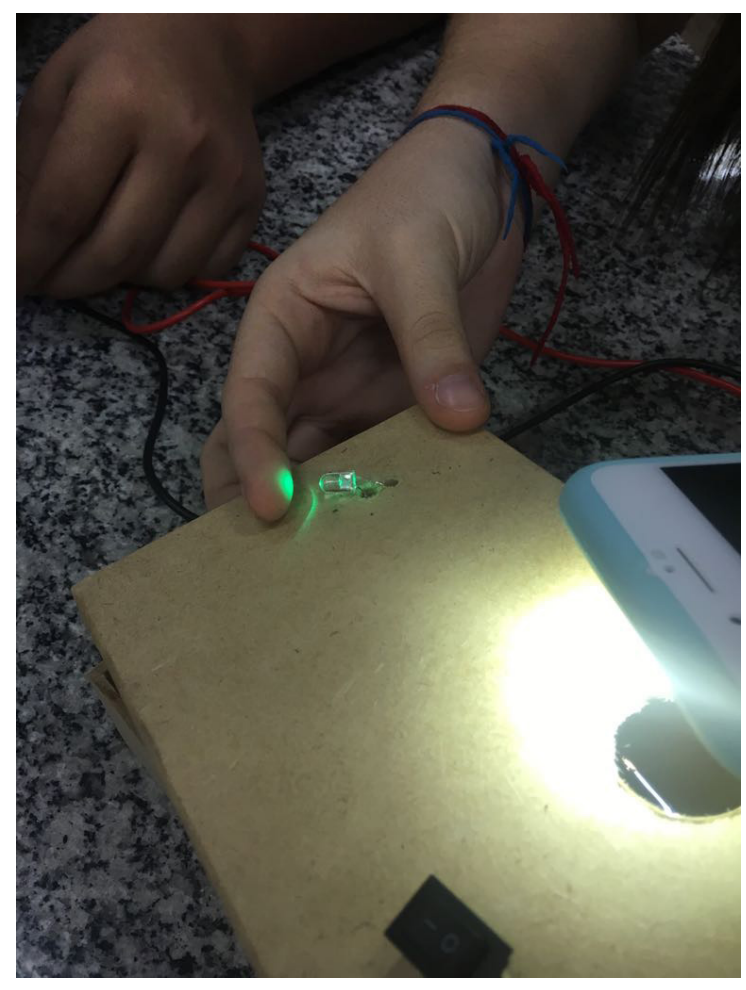

Fonte: dos autores, 2018. 
Resultados e discussões

Os resultados são divididos em duas seções: a obtenção da constante de Planck por método experimental e avaliação diagnóstica de aprendizagem do tema.

1. Obtenção da constante de Planck

Considerando o circuito da Figura 1, os alunos efetuaram as medidas das tensões para cada cor de LED, obtendo-se a seguinte tabela:

Tabela 1 - Medidas das tensões realizadas pelos alunos.

\begin{tabular}{|c|c|c|c|}
\hline \multirow{2}{*}{ GRUPOS } & LEDS & TENSÕES (V) & $1 / \lambda\left(\mathbf{1 0}^{\mathbf{6}} \mathbf{m}^{-1}\right)$ \\
\hline \multirow{3}{*}{ GRUPO 1 } & AMARELO & 1,93 & 1,7316 \\
& VIOLETA & 2,92 & 2,4390 \\
& VERMELHO & 1,80 & 1,4652 \\
\hline \multirow{2}{*}{ GRUPO 2 } & AZUL & 2,62 & 2,2075 \\
\hline \multirow{3}{*}{ GRUPO 3 } & AMARELO & 2,00 & 1,7316 \\
& VIOLETA & 2,90 & 2,4390 \\
& VERMELHO & 1,83 & 1,4652 \\
& AZUL & 2,54 & 2,2075 \\
\hline & AMARELO & 1,96 & 1,7316 \\
& VIOLETA & 2,94 & 2,4390 \\
\hline
\end{tabular}

Com base nos valores obtidos e expostos na Tabela 1, os dados foram tratados usando o programa comercial Origin 8.0, obtendo-se os seguintes gráficos:

Figura 5 - Dados experimentais fornecidos pelo Origin 8.0 do grupo

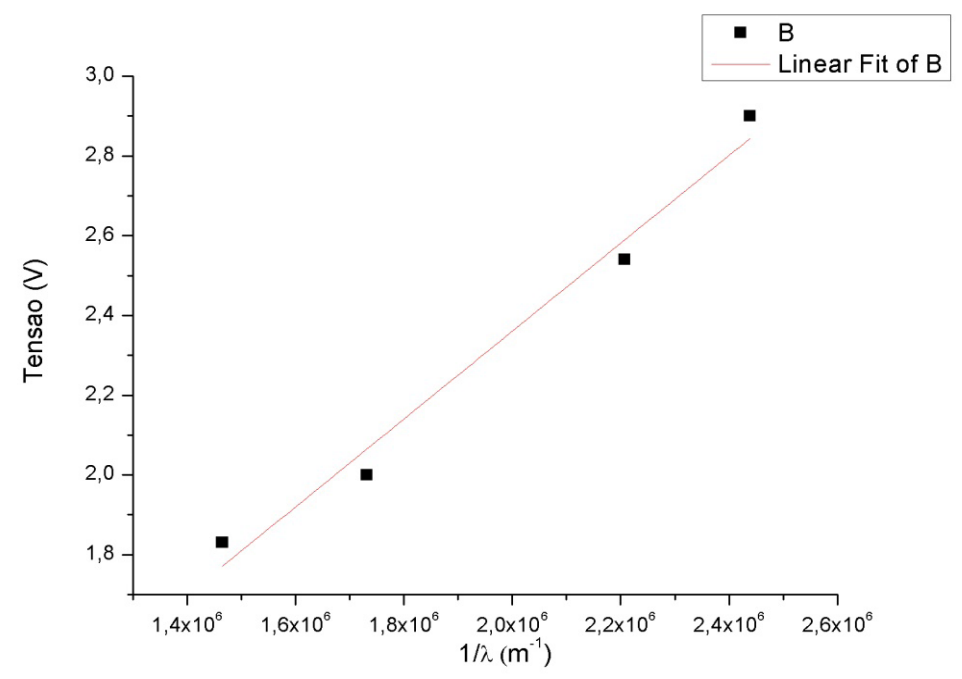


Figura 6 - Dados experimentais fornecidos pelo Origin 8.0 do grupo 2.



Figura 7 - Dados experimentais fornecidos pelo Origin 8.0 do grupo 3.

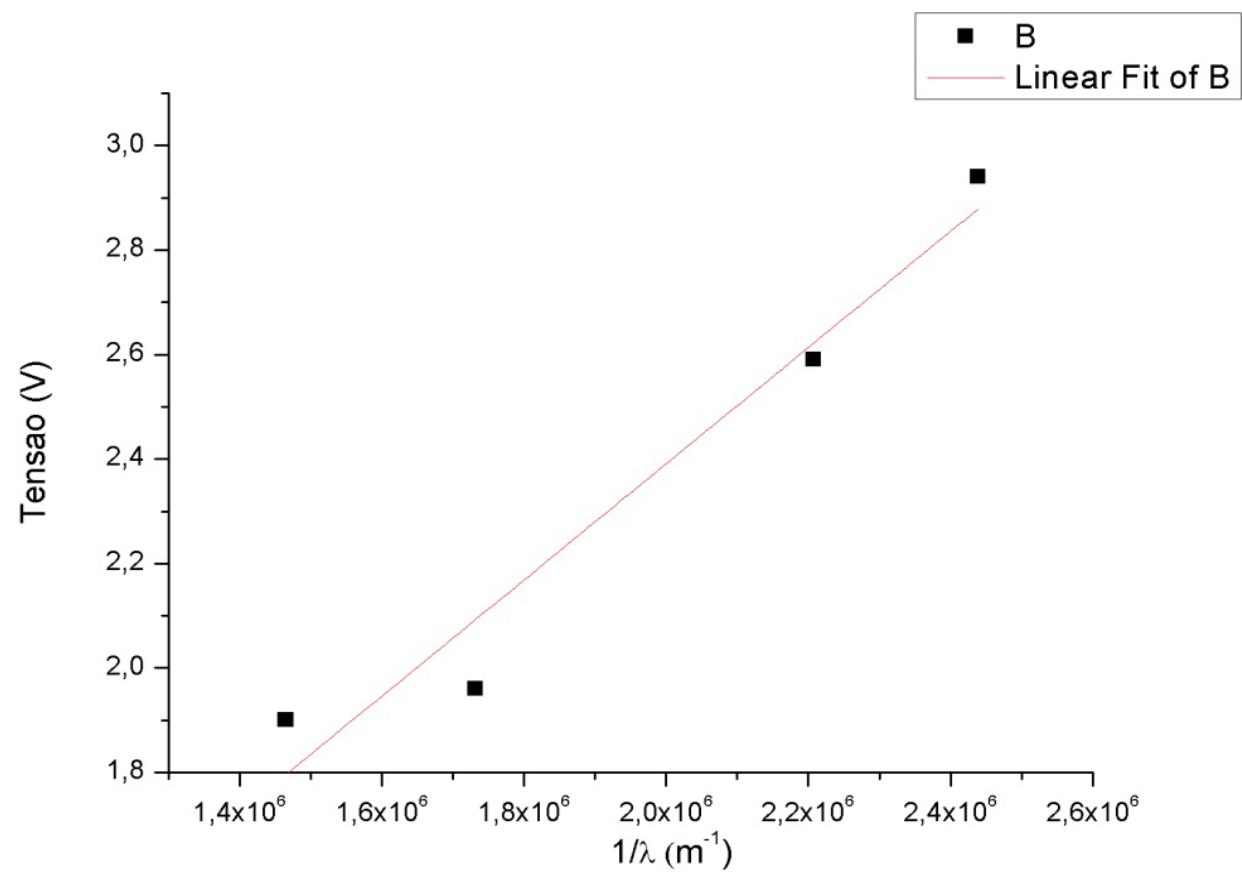


Os coeficientes angulares obtidos com o Origin 8.0 são expressos na Tabela 2:

Tabela 2 - Valores dos coeficientes angulares calculados pelos três grupos de alunos

\begin{tabular}{|c|c|}
\hline GRUPOS & $\begin{array}{c}\text { Coeficiente Angular } \\
\text { (Origin 8.0) }\end{array}$ \\
\hline GRUPO 1 & $1,19 \times 10^{-6}$ \\
\hline GRUPO 2 & $1,10 \times 10^{-6}$ \\
\hline GRUPO 3 & $1,11 \times 10^{-6}$ \\
\hline
\end{tabular}

De posse dos valores da Tabela 2, substituindo esses valores na equação 7, e considerando os valores das constantes $e$ e $c$, é possível obter a constante de Planck. Assim, os valores obtidos por cada grupo foram os seguintes:

Tabela 3 - Resultados obtidos pelos alunos.

\begin{tabular}{|c|c|c|}
\hline GRUPOS & $\begin{array}{c}\text { Constante de Planck } \\
(\mathrm{J.s})\end{array}$ & \begin{tabular}{c} 
Erro Percentual \\
\hline GRUPO 1
\end{tabular} \\
\hline GRUPO 2 & $6,33 \times 10^{-34}$ & $4,4 \%$ \\
\hline GRUPO 3 & $5,88 \times 10^{-34}$ & $11,2 \%$ \\
\hline
\end{tabular}

Comparando-se os valores obtidos na Tabela 3 com o valor da constante de Planck dada na literatura, a qual é 6,62 $\times 10^{-34}$, pode-se perceber que os valores obtidos pelos alunos estão muito próximos do teórico, ou seja, o valor mais próximo (grupo 1) corresponde a cerca de $95 \%$ do valor tabelado na literatura, ao passo que o valor mais distante (grupo 2) chega a aproximadamente $89 \%$ do valor esperado. Possíveis erros estão relacionados a precisão dos equipamentos utilizados e/ou mesmo na montagem dos circuitos. Vale ressaltar que, por questões de segurança, os alunos não soldaram os componentes, o que pode levar a um erro nas medidas.

\section{Avaliação Diagnóstica de aprendizagem do tema}

Para realizarmos a avaliação diagnóstica de aprendizagem do tema proposto neste trabalho, foi elaborada uma lista com 4 questões de vestibulares sobre o tema. ${ }^{6}$ Essa lista foi aplicada aos alunos que participaram desse projeto e para os alunos do $3^{\circ}$ Ano do Colégio Anglo Portal de Limeira. Foram escolhidos, para participarem desta pesquisa, os 11 melhores alunos da disciplina de Física do $3^{\circ}$ ano (fora as duas alunas participantes do projeto) e os 11 alunos participantes da ICJ.

$\overline{{ }^{6} \text { Questões retiradas do }}$ site https://www.sprweb.com.br/mod_superpro/index.php. 
Figura 8 - Comparação de desempenho entre os alunos do terceiro ano do ensino médio, os quais não tiveram contato com o tema proposto, e alunos da ICJ participantes deste projeto.

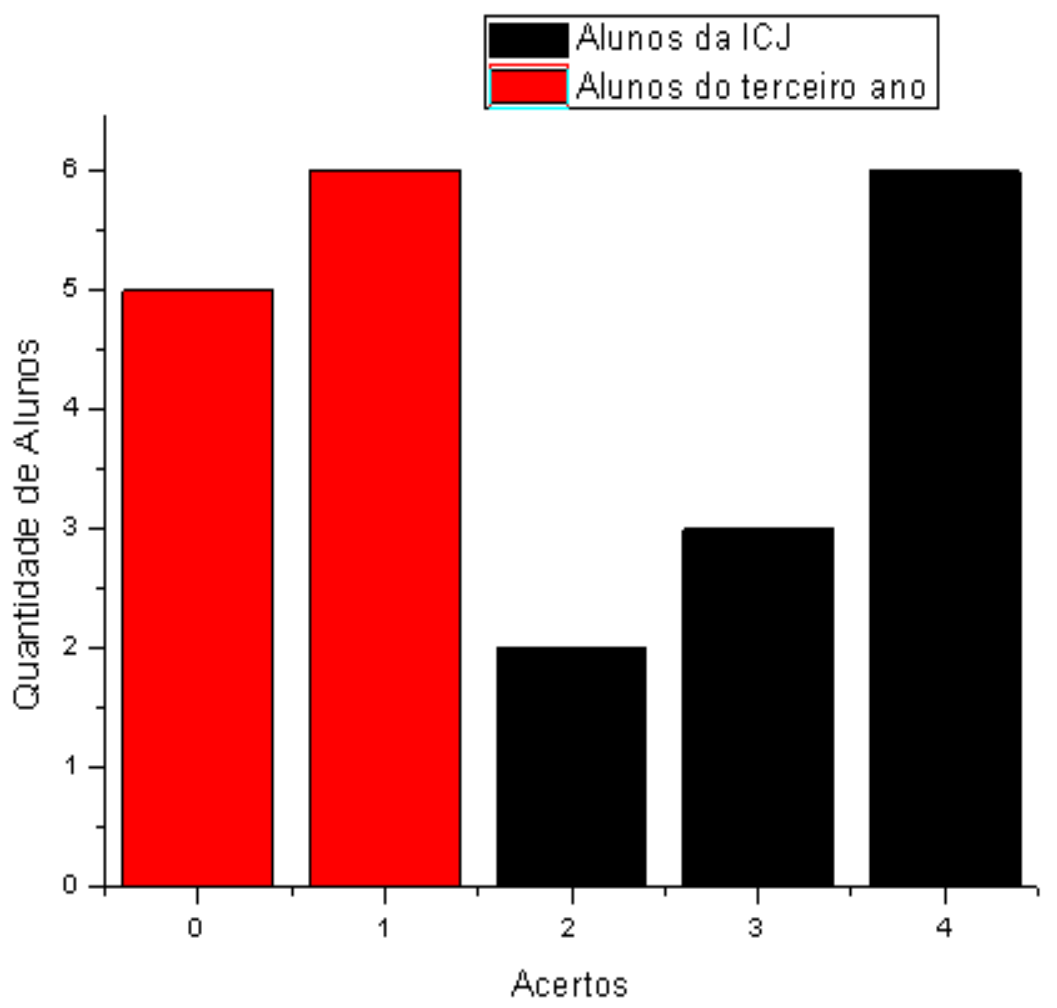

Observando-se o gráfico da Figura 5 é fácil notar que a discrepância de acertos das questões dos alunos que estudaram o tema deste trabalho, através da experimentação, é maior do que aqueles alunos do $3^{\circ}$ ano, ou seja, a maior parte dos alunos participantes da ICJ acertaram as quatro questões propostas, ao passo que os alunos do terceiro ano acertaram duas questões no máximo.

É nítido que mesmo as questões de vestibulares propostas contemplarem apenas a interpretação do enunciado, se os alunos não estudaram um determinado tema, o grau de dificuldade torna-se elevado. Os alunos do terceiro falavam com frequência: "não sei como começar a questão", mesmo o enunciado fornecendo todas as informações necessárias.

A primeira questão, da Universidade Federal de Uberlândia-MG, de 2018, aplicada aos alunos foi a seguinte:

(UFU 2018) As radiações eletromagnéticas possuem diversas aplicabilidades na vida cotidiana, e o espectro das mais utilizadas pela humanidade é formado por radiações que possuem comprimentos de onda que vão desde dimensões atômicas (raios X e radiação gama) até centenas de metros (ondas de rádio). Conforme a ciência atual postula, a radiação eletromagnética possui caráter dual: pode ser considerada partícula ou onda, dependendo da situação em estudo. Pode-se associar a cada feixe de radiação eletromagnética um feixe de partículas chamadas de fótons, e a energia de cada fóton depende de uma constante, chamada de constante de Planck ( $\left.h=6,64 \times 10^{-34} \mathrm{~J} \cdot \mathrm{s}\right)$, e é diretamente proporcional à frequência da radiação. 
Sobre as radiações eletromagnéticas são feitas as seguintes afirmações:

I. Quanto menor o comprimento de onda da radiação eletromagnética maior a energia do fóton a ela associado.

II. Quanto menor a energia de um dado fóton associado a uma dada radiação eletromagnética menor a sua velocidade de propagação.

III. A energia de um feixe eletromagnético constituído de radiação de frequência constante é discreta, ou seja, só pode assumir valores múltiplos inteiros de um valor mínimo.

Em relação às afirmações acima, marque $\mathrm{V}$ para as verdadeiras e $\mathrm{F}$ para as falsas e assinale a alternativa correta.
a) $\mathrm{I}-\mathrm{V}$; II - V; III - F.
b) $\mathrm{I}-\mathrm{V}$; II - F; III - V.
c) I - F; II - V; III - F.
d) I - F; II - F; III - V.

Nesta questão os alunos não foram capazes de associar os temas estudados - como ondulatória, onde o comprimento de onda é inversamente proporcional a frequência - com o tema da equação de Planck. Nota-se que no enunciado foram dadas dicas da equação de Planck, ou seja: "Pode-se associar a cada feixe de radiação eletromagnética um feixe de partículas chamadas de fótons, e a energia de cada fóton depende de uma constante, chamada de constante de Planck $\left(\mathrm{h}=6,64 \times 10^{-34} \mathrm{~J} \cdot \mathrm{s}\right)$, e é diretamente proporcional à frequência da radiação". Assim, os alunos teriam condições de escrever a equação: $\mathrm{E}=\mathrm{hf}$, em que a frequência é diretamente proporcional à energia. A partir dela, na afirmativa I, deveriam lembrar que a frequência é uma grandeza estudada na Física Clássica, ou seja, está associada a

$$
\mathrm{c}=\lambda \mathrm{f} \rightarrow \lambda=\frac{\mathrm{c}}{\mathrm{f}}
$$

Assim, o comprimento de onda $(\lambda)$ é uma grandeza inversamente proporcional à frequência (f). Portanto, a afirmação I estaria correta. Por sua vez, na segunda afirmação, bastava o aluno se lembrar das aulas de óptica, em que os professores falam que a velocidade da luz no vácuo é constante, tornando-a falsa. Por fim, na terceira e última afirmação da questão, observa-se que a energia é dependente do número de fótons, tornando-a verdadeira. Deste modo, a energia depende de um número inteiro de fótons e, portanto, é quantizada, conforme induz a interpretação do enunciado. Em vias gerais nota-se que os alunos não foram capazes de fazer a interpretação do enunciado, passando a uma análise matematizada da fórmula. Também não associaram os conceitos de ondulatória comumente estudados no ensino médio. 
A segunda questão, do vestibular da Universidade Federal de Santa Maria - RS, de 2013:

(UFSM 2013) Uma das características fundamentais das ondas eletromagnéticas, como ocorre em todo o movimento ondulatório, é o transporte de energia. A energia das ondas eletromagnéticas que vêm do Sol é um dos fatores que torna possível a vida na Terra.

A energia de cada fóton da radiação eletromagnética que se percebe como a cor verde pode ser calculada pelo produto da pelo(a) . Essa radiação tem a mesma que qualquer outra onda eletromagnética no vácuo.

Assinale a alternativa que completa as lacunas.

a) frequência - comprimento de onda — velocidade

b) constante de Planck — comprimento de onda - frequência

c) constante de Planck - frequência — velocidade

d) velocidade - massa do fóton - frequência

e) massa do fóton — frequência — velocidade

Esta questão cobra um conhecimento mais direto do tema. Não dá indícios aos alunos sobre o que são os fótons, e tampouco da quantização da energia. Embora ela dependesse de um conhecimento prévio, foi escolhida esta questão de forma sequencial, a fim de verificar novamente se os alunos seriam capazes de fazer associação dela com a primeira questão. Pelo gráfico da Figura 5, percebe-se que a maior parte dos alunos do terceiro ano acertaram somente esta questão. Acreditamos que o fato do enunciado estar mais sucinto facilitou a interpretação dos alunos.

Quando as questões exigem práticas matemáticas, nota-se que os alunos se perdem por completo. De fato, em relação às duas últimas questões, as quais envolviam cálculos, nenhum dos alunos do terceiro ano foi capaz de acertá-la - diferentemente dos alunos da ICJ, os quais, por estarem acostumados com a análise experimental obtida nas medidas, acertaram completamente. Assim, a terceira questão proposta, do vestibular da Universidade Federal do Rio Grande do Sul - RS, de 2011, faz uma avaliação do conhecimento matemático do tema por parte dos alunos:

\section{TEXTO PARA A PRÓXIMA QUESTÃO:}

A nanotecnologia, tão presente nos nossos dias, disseminou o uso do prefixo neno (n) junto a unidades de medida. Assim, comprimentos de onda da luz visível são, modernamente, expressos em nanômetros (nm), sendo $1 \mathrm{~nm}=1 \times 10^{-9} \mathrm{~m}$.

(Considere a velocidade da luz no ar igual a $3 \times 10^{8} \mathrm{~m} / \mathrm{s}$.)

(UFRGS 2011) Cerca de 60 fótons devem atingir a córnea para que o olho humano perceba um flash de luz, e aproximadamente metade deies são absorvidos ou refletidos pelo meio ocular. Em média, apenas 5 dos 
fótons restantes são realmente absorvidos pelos fotorreceptores (bastonetes) na retina, sendo os responsáveis pela percepção luminosa.

$$
\text { (Considere a constante de Planck h igual a } 6,6 \times 10^{-34} \mathrm{~J} \cdot \mathrm{s} \text { ) }
$$

Com base nessas informações, é correto afirmar que, em média, a energia absorvida pelos fotorreceptores quando luz verde com comprimento de onda igual a $500 \mathrm{~nm}$ atinge o olho humano é igual a
a) $3,30 \times 10^{-41} \mathrm{~J}$.
b) $3,96 \times 10^{-33} \mathrm{~J}$.
c) $1,98 \times 10^{-32} \mathrm{~J}$.
d) $3,96 \times 10^{-19} \mathrm{~J}$.
e) $1,98 \times 10^{-18} \mathrm{~J}$.

A resolução adequada é a seguinte:

$$
E=5 h f=5 h \frac{C}{\ddot{e}} \rightarrow E=5 \times 6,6 \times 10^{-34} \frac{3 \times 10^{8}}{500 \times 10^{-9}}=1,98 \times 10^{-18} J
$$

Os alunos não perceberam que o enunciado mencionava a existência de 5 fótons: "Em média, apenas 5 dos fótons restantes são realmente absorvidos pelos fotorreceptores (bastonetes) na retina, sendo os responsáveis pela percepção luminosa". Foi notado, durante a correção, que novamente não perceberam a relação entre a velocidade da luz no vácuo, a frequência e o comprimento de onda da luz.

Na última questão, da Universidade Federal de Goiás - GO, 2005, foi possível notar que os alunos do terceiro ano não conseguiram perceber que bastava fazer a substituição na fórmula (que deveria ser extraída do enunciado da questão 1).

(UFG 2005) A cor amarela característica das lâmpadas de vapor de sódio tem comprimento de onda de 590 $\mathrm{nm}$ e é o resultado de transições eletrônicas do subnível 3p para o subnível 3s do átomo de sódio. Calcule, em elétron-volts, a diferença de energia entre esses subníveis.

Dados:

Velocidade da luz $=300000 \mathrm{~km} / \mathrm{s}$

Constante de Planck $=4,1 \times 10^{-15} \mathrm{eV} . \mathrm{s}$

Esta questão trata de um tema estudado no primeiro ano do ensino médio na disciplina de Química, sobre as transições nos subníveis de energia. A única diferença está na unidade da constante de Planck dada pelo enunciado, ou seja, aqui a constante é expressa em termos de eV.s (eletron-volts). Os alunos da ICJ tiveram contato com essa unidade durante a explicação teórica sobre a forma de conversão entre as leituras de tensões e as respectivas 
energias. Os alunos do terceiro relataram que não haviam compreendido o trecho "é o resultado de transições eletrônicas do subnivel 3p para o subnivel $3 s$ do átomo de sódio". A resolução correta é dada da seguinte forma:

$$
\Delta \mathrm{E}=\frac{\mathrm{hc}}{\lambda} \rightarrow \Delta \mathrm{E}=\frac{4,1 \times 10^{-15} \times 3 \times 10^{8}}{590 \times 10^{-9}} \rightarrow \Delta \mathrm{E}=2,08 \mathrm{eV}
$$

Destaca-se, com essa questão, a importância dos professores de Química fazerem uma associação com a equação de Planck, ao explicarem os subníveis de energia do átomo. De fato, a equação de Planck mostraria aos alunos que os subníveis correspondem, justamente, à definição de quantização.

\section{Percepções dos alunos}

Abaixo são expostas as percepções dos alunos acerca deste projeto:

Aluno 1: "A iniciação científica é uma oportunidade incrível para aprofundar meus conhecimentos em Física moderna e também para entrar em contato com o clima do Ensino Superior. Esse projeto me permitiu novos conhecimentos teóricos e práticos, além de me permitir uma interatividade em grupo com adolescentes de diferentes idades e personalidades. A responsabilidade para com o projeto e pontualidade para a entrega dos resultados das medições foram importantíssimos para o sucesso da iniciação, e são os dois aspectos (responsabilidade e pontualidade) que eu mais desenvolvi ao longo da pesquisa, além de, claro novos conhecimentos. Em uma visão mais ampla, a iniciação cientifica no Ensino Médio é imprescindível para que os jovens valorizem a pesquisa brasileira, já que o contato com ela permite maior entendimento acerca de sua importância e suas capacidades de inovação".

Aluno 2: "A ICJ é um projeto muito interessante. No geral a ideia é muito boa: promove um tipo de estudo que se diferencia dos padrões de ensino, usando aparatos simples e de baixo custo. Incentivar estudos experimentais no Ensino Médio é uma ótima maneira de aumentar o interesse dos alunos na ciência e ajuda a desenvolver um senso mais criativo na resolução de problemas. Lidar com as falhas durante os experimentos e ver o progresso conforme a margem de erro diminui é muito satisfatório e intrigante. Até agora tem sido muito bom poder trabalhar com algo diferente. Creio que muitos profissionais de ensino irão se beneficiar com esse projeto."

Aluno 3: “A Iniciação Científica está proporcionando um valor maior ao meu currículo acadêmico, pois além de nos colocar em contato com pesquisas e descobertas e abrir uma oportunidade para alunos do ensino médio publicarem artigos científicos com seus nomes, essa experiência nos torna mais maduros, tanto de forma pessoal quanto intelectual, e muda nossa visão do futuro, nos mostrando como é importante e enriquecedor a participação em simpósios, a colaboração e trabalho em equipe, a busca por conhecimento, o questionamento. Além de que é em momentos como esse que o aluno tem a oportunidade de por em prática o que foi visto em algum momento nas aulas, podendo então questionar não só a teoria, mas ver como tudo funciona na prática. Toda essa experiência 
abre portas para nós, alunos e participantes de programas como esse, no futuro tornarmos grandes pessoas que farão o diferencial em suas faculdades e trabalhos. Por isso só tenho a agradecer ao professor que nos permitiu que fizéssemos parte de uma Iniciação Científica, a qual pretendo ser apenas a primeira de várias”.

Aluno 4: “A Iniciação Científica Junior está sendo para mim um incrível marco inicial para minha formação acadêmica, nela estou aprendendo diversas coisas novas muito importantes para minha vida que com certeza serão cruciais para o meu futuro, e também descobri o quanto a física é incrível, e não apenas um monte de fórmulas sem sentido, já que usei diversas delas no nosso experimento, e tudo isso graças ao nosso professor Yuri, que trouxe para nossa escola um projeto praticamente inédito no Brasil, e que com certeza mudará nossas vidas"

Aluno 5: "Para mim esse projeto foi uma enorme possibilidade aplicada por nosso professor para que aprendêssemos com a pesquisa a coletar observações de nossos experimentos e escrevermos um artigo científico. Além de adquirir um grande conhecimento com o projeto, abrimos com maior facilidade portas para as universidades no futuro. Vimos também que com um simples experimento podemos descobrir ótimos resultados e mínimos erros. Isso trouxe muita responsabilidade e comprometimento, incentivou o trabalho coletivo e resultou em um fantástico trabalho realizado por todos nós".

Aluno 6: "Para mim, a iniciação científica foi uma experiência essencial, onde pude aprender muitas coisas novas, como trabalhar em equipe para chegar no resultado esperado. É muito bom para o meu currículo ter um artigo publicado estando apenas no primeiro ano do ensino médio e com certeza vai me ajudar muito no futuro. Também foi uma experiência próxima a que vamos ter na faculdade, o que é muito interessante".

Aluno 7: "Para mim com esse projeto sendo realizado, eu obtive mais conhecimento e mais experiência e assim quando eu chegar na faculdade, já vou ter ideia de como pesquisar e alcançar os resultados. Pesquisar é um processo sistemático para ganhar conhecimento e alcançar os objetivos. Com esse projeto, escolas com pouca verba podem faze-lo e ensinar com um preço acessível".

Aluno 8: "Para mim este projeto é muito importante, pois nos proporciona conhecimento além do que se é aprendido na escola e agrega valor aos nossos currículos que podem ser explorados no futuro, com isso aprendi muito, exercemos valores muito importantes como o trabalho em equipe além de abrirmos novos horizontes para o estudo da física, é muito importante a pesquisa no ensino médio pois familiariza os alunos com o que será encontrado no meio acadêmico em uma universidade, fazer pesquisa é descobrir coisas novas é abrir portas para o conhecimento a pesquisa é essencial para o progresso da humanidade por isso devemos implanta-la no ensino médio. A pesquisa não tem faixa etária".

Aluno 9: "A iniciação científica no Ensino Médio está sendo uma grande oportunidade para aprender e ter um pouco de contato com um tipo de atividade universitária. Para os alunos, o fato de podermos participar dessa iniciação trás muito conhecimento, pudemos trabalhar em equipe, aprender mais sobre a constante de Planck e a sobre a fórmula $\mathrm{E}=\mathrm{h}$.f. Além disso temos a oportunidade de em uma média de quinze anos termos nosso nome em um artigo que ajudamos a compor". 
Aluno 10: "Para mim a ICJ foi uma experiência totalmente nova e diferente do que estava acostumado, fazer pesquisas, conferir resultados, testar, etc. A iniciação mudou os meus conceitos sobre a física, me apeguei mais ainda pela matéria que já gostava muito. A iniciação também incentiva a responsabilidade de cada um dos membros".

Aluno 11: “A iniciação Científica foi muito importante para mim, pois consegui abrir uma visão maior para a física, que antigamente achava uma matéria muito complicada, e o projeto me ajudou a perceber o que é a Física Realmente, aprendi também que necessário fazer pesquisas e experimentos práticos mesmo no ensino médio para incentivar a estudar mais e se interessar mais pela a escola e pelo ensino que temos, pude perceber com esse projeto que podemos fazer pesquisas em várias Matérias como história e Geografia, e do tenho a agradecer ao meu professor Yuri que nos incentivou a participar de um projeto relativamente novo no Brasil, e de dar a nos essa possibilidade".

Aluno 12:: "Assim que ingressei ao ensino médio, tenho focado cada vez mais em meus estudos e o que representam para meu futuro. Procuro agarrar todas as oportunidades e não deixar passar nada que possa me ajudar a alcançar os degraus mais altos e mais desejados. Confesso que as ciências exatas me causaram um certo receio. No entanto com as aulas e pesquisas aprendi que nada é impossível, basta querer. Fazer pesquisa para mim só me torna mais seguro e mais confiante, pois através dela encontro novos horizontes e verdades que são só minhas. O projeto de iniciação me trouxe novas descobertas e me fez pensar que eu posso chegar onde eu quiser. Basta trabalhar e ter vontade de vencer. Hoje tenho metas e sigo atrás de meus sonhos".

\section{Considerações Finais}

A Física é uma Ciência fundamentada na Filosofia Natural, ou seja, faz parte do nosso cotidiano. É necessário mostrar, portanto, sua real importância na sociedade. Assim, o tema de Física Moderna é de extrema importância e deve ser, de fato, abordado no Ensino Médio de forma sistemática. Com o desenvolvimento deste projeto, é possível notar que os alunos participantes estão motivados na continuidade de seus estudos, tendo uma visão diferente da Física, conseguindo até mesmo interpretar questões dos vestibulares que tratam do tema proposto neste trabalho. Mesmo com os melhores alunos do terceiro ano que não estudaram a Equação de Planck, percebese que o grau de dificuldade de interpretação é elevado.

Trabalhar um experimento com os alunos do ensino médio nos mesmos moldes de uma iniciação científica de graduação, isto é, seguindo as etapas da metodologia cientifica (estudo das referências, experimentação, debates, análise de resultados, redação de artigo e apresentação em simpósios e congressos), permite que o aluno tenha um envolvimento maior com a disciplina, despertando seu interesse e ajudando-o a perceber que o trabalho da teoria por parte dos professores, em sala de aula, é necessário e tem fundamentos que permitiram o desenvolvimento científico e tecnológico. Este trabalho é forma de fortalecimento do ensino e contribui para a formação de recursos humanos, além de fornecer uma metodologia experimental de baixo custo para o estudo de uma das constantes mais importantes da Física, a constante de Planck. Pode-se dizer, por fim, que os alunos envolvidos adquiriram conhecimento do que é e para que serve fazer pesquisa científica. 


\section{Agradecimentos}

Agradecemos aos mantenedores do Colégio, aos diretores e coordenadores, aos pais e alunos que apoiaram este projeto.

\section{Referências}

AMORIM, M.R. DE; ANDRÉ, S.F.; ANDRÉ, P.S. DE. Classroom fundamentals: measuring the Planck constant. Science in School, Issue 28, p. 28 - 33, 2014.

CÉSAR, R.S.; OLIVEIRA, L.T.; PAIVA, F.M. DE. A constante de Planck: uma forma de ensino de física moderna a partir da experimentação. In: CONEDU - CONGRESSO NACIONAL DE EDUCAÇÃO, 3., 2016. Anais [...]., 2016.

CHICÓRA, T.; CAMARGO, S.; TOPPEL. A. História e filosofia da ciência no ensino de física moderna. In: EDUCERE - CONGRESSO NACIONAL DE EDUCAÇÃO, 12, 2015. Anais [...]., 2015.

MEYER, Y.A. et al. Iniciação Científica no Ensino Médio: a construção de um aparato experimental de baixo custo para estudo da Lei de Lambert-Beer a partir de um circuito montado com fotoresistor LDR. Revista de Estudos Aplicados em Educação, v. 3, n. 5, jan./jun. 2018.

OLIVEIRA, F. F. DE; VIANNA, D.M.; GERBASSI, R.S. Física moderna no ensino médio: o que dizem os professores. Revista Brasileira de Ensino de Física, v. 29, n. 3, p. 447-454, 2007.

\section{ANEXO}

Anexo 1 - Tabela de valores médios dos comprimentos de ondas e frequências do espectro visível.

\begin{tabular}{|c|c|c|}
\hline Cor & Comprimento de onda & Frequência \\
\hline Vermelho & $\sim 625-740 \mathrm{~nm}$ & $\sim 480-405 \mathrm{THz}$ \\
\hline Laranja & $\sim 590-625 \mathrm{~nm}$ & $\sim 510-480 \mathrm{THz}$ \\
\hline Amarelo & $\sim 565-590 \mathrm{~nm}$ & $\sim 530-510 \mathrm{THz}$ \\
\hline Verde & $\sim 500-565 \mathrm{~nm}$ & $\sim 600-530 \mathrm{THz}$ \\
\hline Ciano & $\sim 485-500 \mathrm{~nm}$ & $\sim 620-600 \mathrm{THz}$ \\
\hline Azul & $\sim 440-485 \mathrm{~nm}$ & $\sim 680-620 \mathrm{THz}$ \\
\hline Violeta & $\sim 380-440 \mathrm{~nm}$ & $\sim 790-680 \mathrm{THz}$ \\
\hline
\end{tabular}

Fonte: https://radiacaoblog.wordpress.com/2016/03/11/radiacoes-ultravioleta-visivel-e-infravermelha/. Acessado em 01/09/2018.

Recebido em: 04/12/2018

Aprovado em: 06/05/2019 